\title{
THE LEVEL OF CONTACT LENS PRACTICE IN PORT HARCOURT, RIVERS STATE OF NIGERIA.
}

\author{
BY
}

\author{
AMAECHI*, O. U. AND NWOKEOCHA, E. \\ DEPARTMENT OF OPTOMETRY, FACULTY OF HEALTH SCIENCES, \\ ABIA STATE UNIVERSITY, UTURU, ABIA STATE, NIGERIA \\ EMAIL: okamaechi@yahoo.com \\ *Corresponding author
}

\begin{abstract}
7 he level of contact lens practice in Port Harcourt, Rivers State of Nigeria was investigated. The instruments of research were field work/direct observation, oral interviews of eye care practitioners, and the assessment of clinical records of the contact lens patients from 2003 to 2006. Thirty five eye care practices were studied. The level of contact lens practice was assessed based on the number of clinics offering contact lens services, the number of eye care practitioners involved in contact lens services, the availability of essential instruments required for contact lens care, the percentage of patients prescribed contact lenses, and the annual contact lens patient flow in those clinics. Results showed that eleven (31.43\%) of the eye care practices offer contact lens services, and seventeen (36.96\%) eye care practitioners were involved in contact lens services. The level of contact lens practice based on the availability of retinoscope, trial case, and diagnostic contact lenses was very high (100.00\%), and it was also high based on the availability of slit lamp biomicroscope (72.73\%) and keratometer (63.64\%). The percentage of patients corrected with contact lenses was very low (7.60\%). The annual contact lens patient flow increased from 244 in 2003 to 345 in 2006 (a 41.40\% increase) Enthusiasm on the part of the eye care practitioners in contact lens practice, and creating the proper awareness would boost the level of contact lens practice.
\end{abstract}

KEYWORDS: Contact lenses, contact lens practice, keratometer, refractive correction, slit lamp biomicroscope.

\section{INTRODUCTION}

The advent of contact lenses (CLs) had been credited to Leonardo da Vinci in $1508^{1}$. However development of clinically useful contact lenses has taken place during the working lives of many optometrists now in practice ${ }^{2}$. It is estimated that $50 \%$ of the population utilize some form of refractive correction ${ }^{3}$. Advances in contact lenses and improvements in both materials and designs have resulted in CLs that are applicable for most forms of refractive errors and are both safe and effective for most patients ${ }^{4}$. The desire on the part of the patient to wear contact lenses is one of the most important factors in predicting a successful fitting ${ }^{5}$. A highly motivated patient can often tolerate discomfort and other problems that would be insurmountable to the patient with only superficial motivation ${ }^{6,7}$. Cost and convenience have been implicated as major factors affecting contact lens wear ${ }^{8}$. Contact lenses also come with their complications, which oftentimes are manageable by discontinuing their use. However, more severe, vision threatening complications are less common and include corneal microbial infection ${ }^{9}$, and extreme forms of corneal neovascularization which can lead to scarring of the cornea in the area of the visual axis ${ }^{10}$.

Contact lens practice is yet to attain a significant level in Nigeria. Previous studies ${ }^{11,12,13}$, have shown that contact lens practice in the country is at a low level. The ideal contact lens practice will require the full complement of equipment - keratometer, slit lamp biomicroscope, radiuscope, profile magnifier (shadow graph), complete modification unit, fitting sets and others. Some of these are not readily available in the country, and when available their costs are discouraging. However, the absence of one or more of these does not preclude the fitting of contact lenses. The practice can be as simple as possible to include only the very essential ${ }^{14}$. A simple contact lens practice will require, in addition to other basic instruments required for routine optometric examination- keratometer, slit lamp biomicroscope, retinoscope, trial case, and diagnostic contact lenses. For diagnostic contact lenses, the practitioner can confine himself to just a few lens types ${ }^{15}$. A successful contact lens practice would involve astute practice management. Type of practice to be offered including policy on refund if any, as well as charges should be fully explained 
to the patient and contractual agreement to this effect should be on paper ${ }^{16}$. Our society is becoming more sophisticated so this will help the practitioner in litigation matters. Guarantees should not be given on materials, except where there is one given by the manufacturer. Patient's data gathered during the prefit/fitting/follow-up examinations should be clearly documented to provide complete baseline information for progress evaluation ${ }^{17}$.

Port Harcourt is an oil rich city in Nigeria. Eye clinics are concentrated in Port Harcourt, as is the case in other urban areas in Nigeria ${ }^{18,19,20}$. In this study, the level of contact lens practice in Port Harcourt was determined based on the percentage of clinics that offer contact lens services, the percentage of eye care practitioners involved in contact lens services, the availability of essential instruments required for contact lens practice, the percentage of patients using contact lenses, and the yearly flow of contact lens patients in the clinics studied.

\section{RESEARCH METHODOLOGY}

Thirty five clinics in Port Harcourt metropolis were the focus of the study. Field work/direct observation, oral interviews of the practitioners, and assessment of clinical files were the instruments of research employed. The level of contact lens practice was assessed based on these parameters: the percentage of clinics that offer contact lens services, the percentage of eye care practitioners involved in contact lens services, the availability of essential instruments required for contact lens practice, the percentage of patients who wear contact lenses, and the yearly flow of contact lens patients for the four year period in those clinics.

Field work/direct observation and oral interviews of practitioners were used to establish the number of clinics that offer contact lens services, the number of eye care practitioners involved in contact lens services, and the essential facilities available for contact lens practice in those clinics. The clinical records of practitioners offering contact lens services were reviewed from 2003 to 2006 to determine the number of patients prescribed contact lenses, and the yearly flow of contact lens patients in those clinics.

The level of contact lens practice was ranked thus: $0-20 \%$ as very low, $>20-40 \%$ as low, $>40-$ $60 \%$ as moderate, $>60-80 \%$ as high, and $>80$ $100 \%$ as very high.

\section{RESULTS}

Eleven $(31.43 \%)$ out of the thirty five eye care practices offer contact lens services. The number of eye care practitioners involved in contact lens services was $17(36.96 \%)$ out of 46 eye care practitioners interviewed (some eye care practices had ophthalmologists and more than one optometrist working together).

The availability of the essential instruments required for contact lens practice is shown in table 1. In addition to the basic instruments required for routine optometric care, the instruments used to assess level of contact lens practice were keratometer (63.64\%), slit lamp biomicroscope $(72.73 \%)$, retinoscope $(100.0 \%)$, trial case $(100.0 \%)$ and diagnostic contact lenses (100.0\%). The number of patients given vision correction was 15,333 , and $1,166(7.60 \%)$ were corrected with contact lenses; a ratio of 1 contact lens wearer to 13 spectacle wearers. The yearly patient flow for the 4 year period (2003-2006) is shown in table 2 . There was a gradual yearly increase of contact lens patients, resulting to a total increase of $41.40 \%$.

\section{DISCUSSION}

The level of contact lens practice based on the number of eye care practices that offer contact lens services $(31.43 \%)$, and the number of eye care practitioners that provide contact lens services $(36.96 \%)$ was low. A previous study ${ }^{11}$, stated that not many optometrists are sufficiently interested in, or equipped for this aspect of the profession.

The essential instruments required for contact lens practice were available in the clinics that offer contact lens services (table 1). The level of contact lens practice based on the availability of retinoscope, trial case, and diagnostic contact lenses was very high $(100.0 \%)$. Based on the availability of slit lamp biomicroscope (72.73\%) and keratometer (63.64\%), the level of contact lens practice was high. The superior comfort and ease of adaptability to soft contact lenses have made them to be more frequently used ${ }^{21,22,23,24}$. This could be the reason why the availability of the keratometer recorded that percentage, because soft contact lenses have a specified range of diameters that could fit a large number of patients since it drapes over the cornea, hence the keratometer could become dispensable. However, it is encouraging to observe that the few practices that offer contact lens services are essentially equipped to do so.

The percentage of patients corrected with contact lenses ( $7.60 \%)$ was very low. Out of 15,333 
patients that needed vision correction, only 1,166 were prescribed contact lenses, a ratio of 1 contact lens wearer to 13 spectacle wearers. This low percentage of contact lens wearers was recorded in precious studies ${ }^{12,13,24}$. It has been suggested that with the proper awareness, an increasing number of patients using spectacles would like to use contact lenses ${ }^{12}$. This study ${ }^{12}$ stated that the uncomfortable and uninteresting nature of the prefit/fitting examinations were cited by some patients as reason for preferring spectacles. There was, however, an increase $(41.40 \%)$ in the annual flow of contact lens patients (table 2).

It is recommended that more optometrists rekindle their interest in contact lens practice. With the proper awareness and attitude to practice, more patients would embrace the use of contact lenses.

\section{REFERENCES}

1. Hofstetter, H.W. and Graham, R. (1953): Leonardo and Contact lenses. Am. J. Optom., 30:41-5.

2. Grosvenor, T. P. (1996): Primary care Optometry, $3^{\text {rd }}$ Edn. Butterworth-Heinemann, 665pp.

3. Bennett, I. and Aron, F. (1993): State of the Profession. Optom. Econ, 3:8-14.

4. Barr, J. T. and Bailey, N. J. (1991): The development of Contact lenses In: Bennett, E. S. and Weissman B. A., Edns-Clinical Contact Lens Practice. J. B. Lippincott, Philadelphia, pp1-11.

5. Cole, O. W. (1971): "If contacts fit, why can't I wear them?" Contacto, 15(2): 5-9.

6. Beacher, L. L. (1967): The Psychological Aspects in Contact lens Therapy. J. Am. Optom. Assoc, 38 (3): 185 -9.

7. Paige, N. (1971): Positive Psychological Aspects of Contact lens fitting. Optom. Weekly, 62(17): 378-80.

8. Lee, Y. C., Luin, C. W., Saw, S. M. and Koh, D. (2000): Prevalence and Pattern of Contact lens use in Singapore Community. CLAOJ 26 (1): 21-5.

9. Krachmer, J. H. and Purcell, J. J. (1978): Bacterial Corneal Ulcers in Cosmetic Soft Contact lens Wearers. Arch. Ophthalmol, 96: 57-61.

10. Rozenman, Y., Donnenfeld, E. D. and Cohen, E. J. (1989): Contact Lens related deep Stromal Neovascularization. Am. J. Ophthalmol, 107: 27-32.

11. Ukwade, M. T. (1986): Contact lens Practice in Nigeria. The Cont. lens J, 14 (10):3-5.

12. Ukwade, M. T. (1987): Viability of Contact lenses as a mode of correction in Nigeria. J. Nig. Optom. Assoc, 3 (1): 19-21.
13. Oghre, E. (2004): Trends in Contact lens Practice in Nigeria (1994-2000). AFSCOPE, 1(1): 50-4.

14. Ukwade, M. T. (1985): Improvising for the Nigerian Contact lens Patient: A hypothetical but viable approach. Paper presented at the Annual conference of the Nigerian Optometric Association, Lagos, May 25, 1985.

15. Ukwade, M. T. (1988): Contact lenses: How to Add Pep to your Practice. J. Nig. Optom. Assoc, 4(1): 10-11.

16. Classe, J. G. (1986): Contractual Considerations in Contact lens Practice. J. Am. Optom. Assoc, 57(3): 220-5.

17. Miller, P. J. (1986): Liability Issues in Contact lens Practice. J. Am. Optom. Assoc, 57 (3): $227-9$.

18. Ogbuehi, P. O. (1987): Optometrists in Eye Care delivery in Nigeria. J. Nig. Optom. Assoc, 3:1-3.

19. Odjimogho, E. (2001): Optometry as a Primary Health Care Profession - A Review. J. Optoboard, 1(1): 19-21.

20. Ikonne, E. U., Amaechi, O. U. and UzoDike, E. B. (2006): The Impact of Optometric Services in Imo State, Nigeria. J. Hlth Vis. Sci, 8 (2): 459.

21. Cho, P. and Conway, R. (1990): Contact lens Practice in Hongkong. Clin. Exp. Optom, 73 (5): 143-50.

22. Morgan, P. and Efron, F. (2001): Trends in UK contact lens Prescribing. J. Am. Acad. Optom. Supp. Optom. Vis. Sci, 78 (12): 52-6.

23. Lazon, P. (1996): Contact lens Practice in Peru. Int. Cont. lens Clin, 23: 44-5.

24. Amaechi, O. U. and Ike O. O. (2008): An Appraisal of Contact lens Wearers in some Major Towns in the Southern Part of Nigeria. J. Nig. Optom. Assoc, 14:3-6. 
TABLE 1: ESSENTIAL INSTRUMENTS AVAILABLE FOR CONTACT LENS PRACTICE

\begin{tabular}{|l|l|}
\hline Instruments & Number of Clinics (\%) \\
\hline Keratometer & $7(63.64 \%)$ \\
Slit Lamp Biomicroscope & $8(72.73 \%)$ \\
Retinoscope & $11(100.0 \%)$ \\
Trial Case & $11(100.0 \%)$ \\
Diagnostic Contact Lenses & $11(100.0 \%)$ \\
\hline
\end{tabular}

TABLE 2: ANNUAL CONTACT LENS PATIENT FLOW FROM 2003 TO 2006

\begin{tabular}{|l|l|l|l|l|l|}
\hline Year & $\mathbf{2 0 0 3}$ & $\mathbf{2 0 0 4}$ & $\mathbf{2 0 0 5}$ & $\mathbf{2 0 0 6}$ & Total \\
\hline No of patients & 244 & 258 & 319 & 345 & 1,166 \\
Annual Percentage Increase & - & $5.74 \%$ & $25.0 \%$ & $10.0 \%$ & $41.40 \%$ \\
\hline
\end{tabular}

